\title{
Using Physics Lab Tours For Pre-College Students To Promote Scientific Identity
}

\author{
Robert D. Niederriter*, Kathleen Hinko*† \\ *Department of Physics, University of Colorado, 390 UCB, Boulder, CO 80309 \\ $†$ JILA, NIST and University of Colorado, 440 UCB, Boulder, CO 80309
}

\begin{abstract}
Drawing on the NAS/NRC goals of identity and engagement, we construct a framework for promoting scientific identity through field trips and lab tours. Design of lab tours for the Partnerships for Informal Science Education in the Community (PISEC) program is presented as an example of applying this framework. We evaluate the success of PISEC's redesigned field trips and lab tours based on observations and analysis of students' questions during lab tours. Students' dialogue during lab tours seeks to know what scientists do and who scientists are, aligning with our goals and with the identity framework.
\end{abstract}

Keywords: physics education, informal learning, lab tours, field trips, identity, children

PACS: 01.40.-d, 01.40.gb

\section{INTRODUCTION}

The lack of diversity in science, technology, engineering and mathematics (STEM), and especially in physics [1,2], is a serious issue for the nation in terms of economic and social equity, as well a major hindrance to advancing the range of perspectives in the disciplines themselves [3]. Strategies to promote greater diversity in STEM include the expansion of access to K-12 informal learning, as these types of environments can provide opportunities to connect community cultures to science [4]. Furthermore, research has shown that students are more likely to persist in STEM when they are able to develop strong science identities [5], which is an explicit goal of informal STEM activities [6].

In this paper, we present a framework for fostering scientific identity in young students through field trips to physics departments and laboratory facilities. Through the lens of scientific identity, we describe learning goals for field trips and specific design principles for lab tours. We describe the application of this framework to a typical field trip to the Department of Physics at the University of Colorado (CU) Boulder. To demonstrate how to evaluate these events with respect to the framework for promoting identity, we discuss observations from field trips and also analyze the students' questions during lab tours. Many physics research institutions and individual physicists engage the community by giving tours of facilities, and we intend this framework to be useful as a model for the design and evaluation of such programs.

\section{PHYSICS IDENTITY IN INFORMAL ENVIRONMENTS}

The National Academy of Sciences' National Research Council (NAS/NRC) describes two learning goals that are explicit in informal science: 1) encouraging "excitement, interest, and motivation" and 2) fostering "identity in learners as someone who knows about, uses, and sometimes contributes to science" [6]. More specifically, Carlone and Johnson, in their interviews with women scientists of color, determined three overlapping aspects to identity: performance, competence, and recognition [7]. Performance is the belief in one's ability to perform required science tasks. Competence is belief in one's ability to understand science content. Recognition happens when others see you as a good science student. Applying this work to college students, Hazari, et al. included the category of interest (as also declared by NAS/NRC), to describe the developing science identity of undergraduate students [5]. Interest is the desire to think about and understand science.

There is considerable literature showing informal STEM programs can promote science identity. For instance, Falk argues that individuals' identities affect their science museum experience [8]. Additionally, Calabrese-Barton has studied the science identity of homeless children in afterschool programs [9].

Since 2007, the CU Boulder Department of Physics and JILA (a joint institute between CU and NIST) have facilitated an informal physics program designed to promote identity, called Partnerships for Informal Science Education in the Community (PISEC) [10]. PISEC consists of two components: 1) weekly, 
afterschool sessions where students do hands-on activities with university physics mentors, and 2) a culminating field trip to $\mathrm{CU}$ where students tour physics laboratories. Through these activities, the PISEC program aims to develop students' science identity across the four categories (performance, competence, recognition, interest).

One particular aspect of the PISEC afterschool program is its reliance on physics graduate and undergraduate volunteers, collectively referred to as University Educators (UEs). Over the course of weekly, afterschool sessions during the semester, the UEs form bonds with the K-8 students as they work together to explore engaging, hands-on physics activities. Touring the laboratories of the UEs at JILA and the CU Physics Department is one of the primary activities on the field trip at the end of the semester. While we have previously studied the afterschool program $[11,12]$, in this paper we focus on the design and evaluation of the field trip and lab tour.

\section{FRAMEWORK FOR PROMOTING IDENTITY}

We argue that an intentionally designed field trip experience can promote the growth of students' science identity, as well as provide opportunities for students to situate their science identity in a physics researcher context. In order to achieve this identity development, we present a framework consisting of 5 goals for physics field trips:

1. Provide resources to encourage students to envision themselves as scientists by demonstrating what it means to be a scientist and making the activity of scientists doing science concrete.

2. Provide resources to encourage students to envision themselves as college students.

3. Emphasize to students that they are scientists (and specifically, that they have been doing real science in the PISEC afterschool program).

4. Show students that science is fun and exciting.

5. Expose students to the culture of scientists (and expose scientists to the culture of the students).

Goals 1, 2, and 5 are aligned with the performance and competence aspects of identity. Goal 3 is related to recognition and Goal 4 encourages interest in science. In Goal 5, the idea to expose scientists to the culture of students demonstrates the possibility for additional goals to be developed that aim to impact those physicists facilitating the field trips and lab tours.

\section{Physics Lab Tour Design}

There are numerous ways in which this framework could be made manifest in field trip design for precollege students. In university or national lab settings, physicists may be responsible for part or all of students' field trip time. Typically, though physicists lead or control the content and format of tours of laboratories and other research-related facilities.

For the PISEC program, we have applied this framework to the both field trips and lab tours. Here we describe lab tour design principles based on the goals of promoting identity growth in the context of the specific nature of our educational program and its participants. For instance, cultural depictions of scientists lead to students to associate scientists with lab coats, test tubes, and Einstein-ian hairdos [13]. K-8 students, especially from traditionally underrepresented groups, are likely unfamiliar with the research activities of physicists or the environment of a research laboratory. Therefore, in alignment with Goals 1 and 5, we seek to demonstrate to students what it means to perform and show competence as a physicist in a lab.

Three major aspects of lab tour design are:

Students will see and hear a UE in a laboratory.

Many students in PISEC have not personally known a scientist nor have they been in a research laboratory before their experience in PISEC. When students come to campus for a lab tour, they are able to see and hear their UE in a different context than the afterschool setting. Being in a laboratory reemphasizes to the student that the UE is in fact a professional scientist. The experience helps students understand what this scientist does in their time outside of PISEC and how the scientist's work in physics is analogous to the work done by the participants in PISEC. After a lab tour, students have a visual image of a real laboratory and can imagine working in that environment.

Students will hear about current science research.

Being exposed to current science research projects helps students recognize what it means to be a scientist today. During the lab tour, UEs explain their research using terms and analogies that are appropriate to the K8 student audience. Students are exposed to topics of interest in active physics laboratories and laboratory equipment. They may be confronted with the idea that doing science in a physics laboratory is a different experience from doing science in their classrooms. UEs address, however, how the content of PISEC activities (circuits, optics, magnets, etc.) connects to ideas or equipment in the lab. By explicitly referencing the similarities between PISEC and lab activities, we aim to reinforce the idea that students are doing science in the PISEC program and that they are scientists. 
Students will touch and interact with objects in the lab.

When students enter a lab for the first time they may be overwhelmed by the sheer amount of objects with which they are unfamiliar. Simple physical interaction with objects present in the lab makes the activities of doing science in that setting more concrete and accessible to the students.

\section{EVALUATION OF IDENTITY}

For programs designed to cultivate students' physics identity, it is important to evaluate the program based on a measure of identity. Two ways we evaluated our program were broad observation followed by more focused/fine-grained analysis: first observing the field trips as a whole, and second analyzing the questions asked by students during lab tours. These methods provide complementary information about the immediate effects of our program on students. The observations indicate whether or not we are actually doing what we intend while the question analysis informs us about what the students think about the experience.

\section{Field Trip Observations}

We observed what actually happened during these events in order to understand in what ways our program was achieving its goals for students. This section describes a field trip from the Spring 2013 semester for a group of $306-8^{\text {th }}$ grade students from two different middle schools. Approximately $2 / 3$ of the students were from groups underrepresented in the sciences. The students and activities described here are representative of other end-of-semester field trips.

The field trip was divided into 4 main parts: lab tours, movies/awards, demos, and a scientist panel. There were several other small activities including a long walk through campus to and from the school bus, but we focus on only the primary sections in this work.

For this field trip, students toured a laser physics laboratory, hosted by a UE; they visited the JILA machine shop with a veteran machinist; and they ate lunch while watching a 3-minute video-recorded lab tour. During the laser lab tour, students saw large physics apparatus, listened to explanations of x-rays, lasers, and the use of computers in the lab, and were able to interact with lab equipment, including donning shoe covers before entering the lab, trying on laser safety glasses, and pushing a button to turn on a laser.

After the tours, the students gathered in a physics lecture hall. Animation movies about science experiments made by the students during the afterschool sessions were projected on the lecture screen, played, and applauded. Students then received participation certificates and awards for their work throughout the semester. At the end of the field trip, UEs made liquid nitrogen ice cream for the students and performed demonstrations using liquid nitrogen. The students froze and smashed flowers and tortillas with liquid nitrogen and ate ice cream.

Another feature was the Scientist Panel, which was comprised of UEs who answered questions from the students about why they chose to study science, their goals for the future, and personal hobbies. There are many similarities between questions asked during the panel and during lab tours, as discussed in the following section.

Many of these activities are designed to impact physics identity. The aspects of identity aligned with each of the field trip activities are listed in Table 1.

TABLE 1: Aspects of physics identity field trips.

\begin{tabular}{c|l}
\hline Activity & Aspects of physics identity \\
\hline Lab tour & Performance/Competence \& Interest \\
\hline Awards & Recognition \\
\hline Demos & Interest \\
\hline Panel & Performance/Competence \& Interest \\
\hline
\end{tabular}

The lab tours brought students into the daily activities of scientists and allowed students to experience the laboratory environment. In the example above, the UE demonstrated what it means to know about, use, and contribute to physics, as well as explaining his own interest in physics. Through certificates and awards, we provide positive recognition of students' work and effort that cultivates the recognition component of identity. We know from prior field trips that many students remember the fun experiences and ask about liquid nitrogen and ice cream the following semesters. By finishing with fun, exciting, and memorable activities, we build interest in physics that will feed into students' physics identity. These observations suggest that the field trip promoted physics identity among the students.

\section{Questions during Lab Tours}

Analyzing the questions asked by students provides another means for evaluating the effectiveness of our field trips. We are interested in the way the students perceive the laboratory spaces and the scientists leading the tours, because these perceptions are directly related to the goals outlined above. The questions they ask and the discussions that follow show how the students engage with the objects, ideas, and people in the lab. We have video-recorded two lab tours and have observed the questions asked during three others. These questions were compiled into a list in order to compare and look for patterns. Upon 
analyzing the list, we found that most student questions fell into 4 broad categories, listed in Table 2 . We do not intend these categories to be exhaustive, but to provide insight to student engagement during lab tours.

TABLE 2: Lab tour question categories.

\begin{tabular}{l|c}
\hline Questions asked on lab tours & $\begin{array}{c}\text { Framework } \\
\text { Goals }\end{array}$ \\
\hline $\begin{array}{l}\text { What are these things in the lab, } \\
\text { and how does this all work? }\end{array}$ & 1,4 \\
\hline $\begin{array}{l}\text { How do these things relate to me } \\
\text { and my life? }\end{array}$ & 3 \\
\hline Who are these people in the lab? & $1,2,5$ \\
\hline $\begin{array}{l}\text { Are these people like cultural } \\
\text { depictions of scientists? }\end{array}$ & 5 \\
\hline
\end{tabular}

The first two categories of student questions relate to the objects, methods, and research ideas seen and heard during the lab tour. The students are interested in the tools and equipment they see around them, and asked questions including, "What's that silver thing?" and "What's that made of?" They pointed to things and actively tried to make sense of their surroundings. These questions indicate that students want to know about the lab and how it is used. Students' questions suggest that the lab tours make the activities of scientists doing science concrete, aligning with Goal 1.

In addition to asking about the equipment around them, students asked about why things are done in a certain way. Questions included: "Why are you wearing Crocs?" (Answer: for reducing static electricity), and "What's the purpose of [your experiment]?" Speculative questions were also common, such as "What would happen if you made a tiny bubble?" or "What would happen if someone made a radioactive volcano?" Students frequently commented during lab tours about how excited they were. Phrases like "this is so cool!" were common, suggesting the tours support students' interest as an aspect of their science identity and are aligned with Goal 4. Students also asked questions that attempted to relate the scientific apparatus in the lab to their everyday lives, which relates to Goal 3. For example, students asked "Where do you find Teflon in everyday life?" and "Is this [glass-blowing] how most glass shapes are formed?" while touring the machine shop.

While the first two categories of questions indicate student curiosity about the objects surrounding them and even the ideas they hear about, many other questions were directed toward understanding the scientists themselves. Students asked questions such as "Is that your favorite T-shirt?" or "How many tattoos do you have?" Some students asked the UEs if they use a science notebook the same way the students record their experiments in notebooks afterschool. They seemed intrigued by the scientists standing in front of them and curious about what it means to be a scientist. Although most of the people giving the tours are UEs whom the students have already met during the afterschool program, the lab environment provokes new questions by reaffirming the identity of the UEs as physicists. These questions indicate that Goals 1, 2, \& 5 are being achieved: students are introduced to the physics community and may be starting to imagine themselves in labs and at college.

An interesting observation was that many of the students' questions and discussion involved references to popular culture science and scientists. For example, students asked about making people radioactive and explained that Superman was radioactive. The students seemed to try to reconcile their prior conceptions of laboratory scientists and the real scientists on the tour.

\section{CONCLUSIONS}

We have provided a framework for cultivating science identity through field trips and lab tours. To demonstrate the framework, we described the design and evaluation of PISEC field trips and lab tours. We observed that students' discussions during lab tours align with our goals and with the identity framework. Other institutions can use these design principles to focus field trips and lab tours towards cultivating physics identity.

\section{ACKNOWLEDGMENTS}

The authors acknowledge the JILA Physics Frontier Center and the CU PER Group, especially Noah Finkelstein and Rosemary Wulf for helpful discussions. RDN is supported by the National Defense Science and Engineering Graduate Fellowship. This work is funded by NSF Grant \#1125844.

\section{REFERENCES}

1. J. Ferrini-Mundy, Science 340, 278 (2013).

2. AIP Report, Women in Physics and Astronomy (2005).

3. T. Hodapp, Interactions, June/July, pp. 29 (2007).

4. NAS, NAE, Institute of Medicine, Expanding Underrepresented Minority Participation. NAP (2011).

5. Z. Hazari et al., J. Res. Sci. Teach. 47, 978 (2010).

6. National Research Council, Learning Science in Informal Environments, National Academies Press, 2009.

7. H. Carlone et al., J. Res. Sci. Teach. 44, 1187 (2010).

8. J. Falk, Identity \& the Museum Visitor Experience, 2009.

9. A. Calabrese Barton, J.Res. Sci. Teach.,35, pp379 (1998).

10. PISEC: http://www.colorado.edu/physics/PISEC

11. R. Wulf, K. Hinko and N. Finkelstein, PERC Proceedings, AIP Press (2012).

12. J. Bartley, L. Mayhew and N. Finkelstein, PERC Proceedings, AIP Press, pp. 93-96 (2009).

13. K. Finson, Sch. Sci. and Math., 102(7) pp 335 (2002). 